\title{
UniverSelf, Realizing Autonomics for Future Networks
}

\author{
Laurent Ciavaglia \\ Alcatel-Lucent \\ Laurent.Ciavaglia@alcatel-lucent.com
}

\begin{abstract}
Despite undeniable progress in the field of autonomic networking, the need for techniques enabling the transformation of operational models, and the evolution towards more flexible networks, real-world deployments of selfmanaging networks are still limited. Most efforts have been focused on solving manageability bottlenecks in a given technological domain, while communication services extend anywhere, regardless of the technological boundaries (e.g. wireline/wireless). The FP7 UniverSelf project is developing an end-to-end and modular framework based common management substrates and network empowerment mechanisms, following a reference trust assurance process to foster large-scale deployment and adoption by the ICT actors.
\end{abstract}

\section{Four Challenging Objectives}

UniverSelf is an FP7 IP project that addresses autonomic networks and the interoperability of their parts in a holistic manner, i.e. including both wireline and wireless parts of the network. This project is vital because the operational complexity in an operator's network is growing, because the cost structure of the current management model is not sustainable, and because the already existing management architecture is no longer adapted. Correspondingly, the four main objectives of UniverSelf are:

- Design a Unified Management Framework for the different existing and emerging architectures that is cross-technology (i.e. wireless and wireline) and will serve as a common platform for both systems and services.

- Design the functions that will enable self-managing networks and embed these functions directly within the systems and elements that comprise the network infrastructure and support service delivery.

- Demonstrating the potential for deployment of autonomic solutions in carrier grade networks with an eye towards stimulating further research in Europe towards application and commercialization.

- Generate confidence in the viability and use of autonomic technologies in telecommunication networks by defining "certification" parameters for autonomic networking products.

\section{$2 \quad$ An Impactful Experimentation Strategy}

UniverSelf aims at demonstrating the feasibility and efficiency of the project solutions, mechanisms and algorithms in a proof-of-concept environment comprising 
simulation modules and prototyping activities, together with testing and assessment capabilities. The experiments are organized according to the timeline shown in Figure1. The validation part of this activity addresses feasibility aspects, as well as assessment of key performance or stability, scalability etc. indicators and works especially on the integration of the solutions in and via the Unified Management Framework (UMF). Experimentation can be seen as a concrete methodology for the production of validation results close to real life scenarios. Experimentation and validation activities focus on the collection and analysis of metrics related to the achieved performance and optimization benefits, QoS in service provisioning, the end-to-end coherence of management actions, the system's stability and responsiveness, the realized compliance to the imposed policies and profiles, the CAPEX and OPEX gains

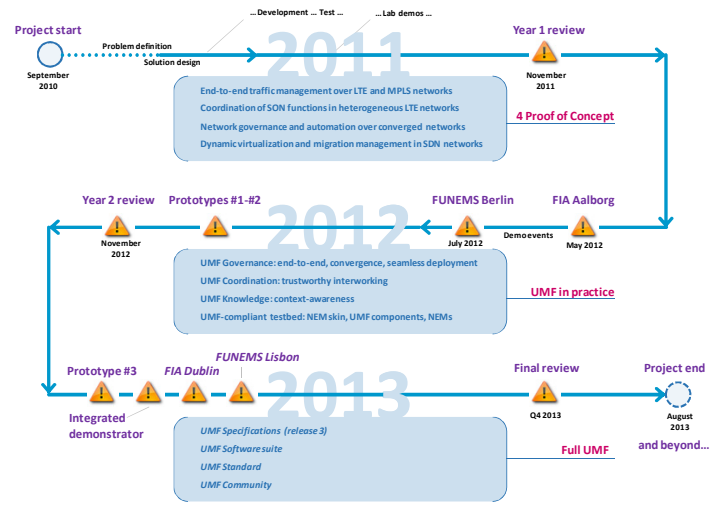

Fig. 1. UniverSelf experimentation timeline etc... The main capabilities targeted for the federated framework for the experimental facilities, are the computation resources, the incorporation of heterogeneous wired and wireless systems and network management operations (emphasis on the autonomic aspects). Moreover, issues related to the business sustainability and user acceptance and trust in autonomic solutions are also addressed.

\section{An Integrated Portfolio}

The aim of the project portfolio is to provide a comprehensive and consistent view about the solutions and technologies developed within the UniverSelf project, their constituting elements, their relationships and respective development levels. The portfolio presents an overall and integrated view about the solutions. The project portfolio is a tool useful to show the industry impact, feasibility, and relevance. The portfolio is structured around three dimensions: the capability levels (of a NEM or core mechanism), the development lifecycle, and the application domain(s). The UniverSelf project produces two essential pieces of solutions tightly related: the Unified Management Framework (UMF) and the Network Empowerment Mechanisms (NEM). These elements constitute the base of the project portfolio. The various combinations (or packaging) of these elements (UMF core functions and mechanisms, NEMs) constitute the project integrated solutions. As example, UMF core functions, mechanisms and specific NEMs are combined together to provide an integrated solution to the use case 1 on self-diagnosis and self-healing for VoIP and VPN services. Similar examples exist for the other project use cases, and infinity of variations can be imagined to address the different problems and use cases. 
The solutions developed are modular, composable, extensible, interoperable, and can interwork with legacy systems. The IT/telco environments where they can be deployed have been evaluated, tested, and benchmarked, Examples of interworking and deployment (options) are also described as part of the project portfolio documentation together with other elements (or views) related to the different solutions such are the applicable business reference use case(s), problems...

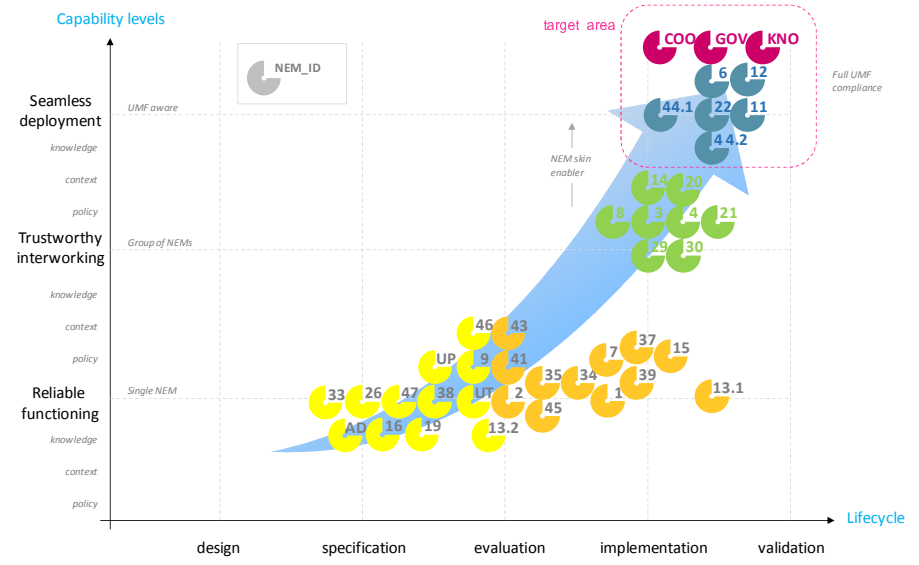

Fig. 2. UniverSelf portfolio (Spring 2013)

\section{The Unified Management Framework (UMF)}

A NEM achieves a self-management function (a closed control loop), with a specific purpose: an operational problem to solve with a defined performance objective in specific technological domain. Examples are for instance the use of Bayesian inference for fault diagnosis in FTTH environments, or the use of genetic algorithm for interference coordination in LTE networks.

When a NEM is deployed within an operator infrastructure, it has to deal with its environment composed by the operator, the network/service equipments, the legacy management systems and also the other NEMs. To realize a seamless deployment and trustworthy interworking of a large number of NEMs, we need a unified framework to manage them. This UMF shall be composed by:

- Tools to deploy, drive and track progress of NEMs which highlight the need for Governance/Human-to-Network tools.

- Tools to avoid conflicts ensure stability and performance when several NEMs are concurrently working which highlight the need for Coordination/Orchestration mechanisms.

- Tools to make NEMs find, formulate and share relevant information to enable or improve their functioning which highlight the need for Knowledge management.

- Tools to allow NEMs getting monitoring data and enforcing configuration actions at equipment level which highlight the need for specific adaptors.

These areas outline the core functional decomposition of the Unified Management Framework, as depicted in Figure 3. 


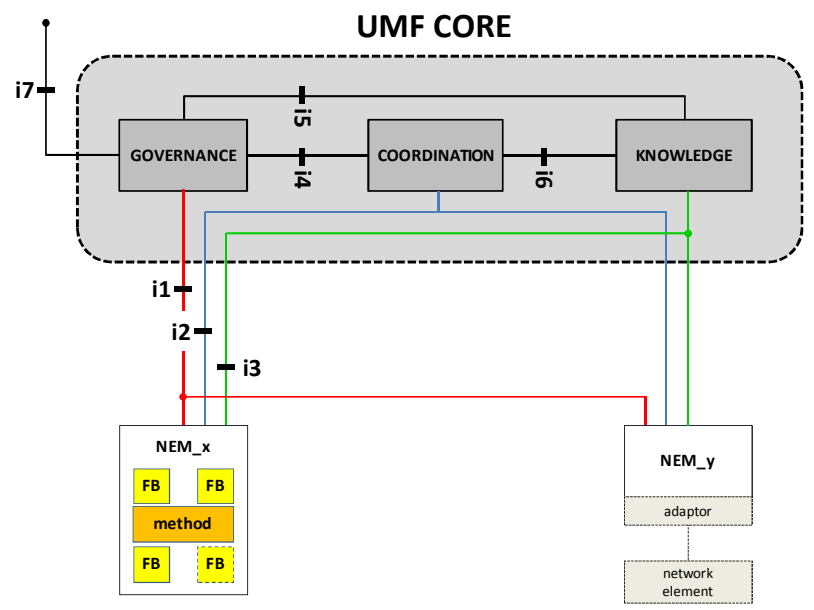

Fig. 3. Functional view of the Unified Management Framework

\section{$5 \quad$ Summary of Main Achievements}

The main achievement the UniverSelf project is the design, specification, demonstration and standardization a Unified Management Framework (UMF) capable of managing any kind of intelligent/autonomic functions over heterogeneous network technologies. The UMF provides operators and vendors with inter-operable (standardized), reliable and trustworthy (tested and certified), seamlessly deployable (unified, end-to-end, and cross-technology) autonomic functions (NEM - Network Empowerment Mechanisms). The UMF CORE is composed of 3 main functional blocks: GOVERNANCE, COORDINATION and KNOWLEDGE designed to manage the large number of NEMs, by providing:

The project provides both written specifications (to be channeled into standards) and readily available and validated software solutions (UMF software/libraries, NEM skin as an accelerator for NEM developers to make them UMF-compliant to support operators and vendors in the development and deployment of these new autonomic functions. In the time frame of the project, three releases of the UMF are/will be developed (currently 3rd release in under specs/development) and multiple autonomic functions (35+) are also specified, developed, tested and validated in real-world conditions. These functions covers both fixed and wireless technologies like several 3GPP/LTE SON functions, IP/MPLS traffic engineering, software-defined networks, learning and prediction algorithms, diagnosis, cloud mobility... The project also demonstrates its solutions on the basis of 6 use cases (based on problems and technologies identified by operators participating in the project (Orange, Telefonica, Telecom Italia, NTT)).

The project results are available on the UniverSelf project website at http://www.univerself-project.eu

Open Access. This article is distributed under the terms of the Creative Commons Attribution Noncommercial License which permits any noncommercial use, distribution, and reproduction in any medium, provided the original author(s) and source are credited. 